\title{
Curriculum Development of a Research Laboratory Methodology Course for Complementary and Integrative Medicine Students
}

\author{
Nicole Vasilevsky ${ }^{1,}{ }^{*}$, Morgan Schafer $^{1}$, Deanne Tibbitts ${ }^{1}$, Kirsten Wright $^{1}$, and Heather \\ Zwickey ${ }^{1}$ \\ ${ }^{1}$ Helfgott Research Institute, National College of Natural Medicine, 049 SW Porter Street, \\ Portland, OR 97201
}

\begin{abstract}
Training in fundamental laboratory methodologies is valuable to medical students because it enables them to understand the published literature, critically evaluate clinical studies, and make informed decisions regarding patient care. It also prepares them for research opportunities that may complement their medical practice. The National College of Natural Medicine's (NCNM) Master of Science in Integrative Medicine Research (MSiMR) program has developed an Introduction to Laboratory Methods course. The objective of the course it to train clinical students how to perform basic laboratory skills, analyze and manage data, and judiciously assess biomedical studies. Here we describe the course development and implementation as it applies to complementary and integrative medicine students.
\end{abstract}

\section{Keywords}

Lab methods; course development; complementary and alternative medicine; research

\section{Introduction}

Complementary and integrative medicine (CIM) is a growing field of healthcare and research that treats disease by using naturally derived interventions to stimulate the body's innate healing ability [1]. Due to an increasing demand for evidence-based clinical interventions, there are growing efforts to integrate research literacy and evidence-based medicine into CIM training programs and clinical practice [2-5]. At the National College of Natural Medicine (NCNM), in Portland, OR, USA, the Helfgott Research Institute (Helfgott) fulfills this role.

The central mission of Helfgott is to advance the science of natural medicine through the conduct of rigorous research studies. However, because it is located at one of the country's leading naturopathic and Chinese medicine schools, Helfgott also provides research training for NCNM students and postdoctoral fellows in CIM and integrative medicine research. In 2011, the Master of Science in Integrative Medicine Research (MSiMR) program was launched at NCNM and Helfgott to provide formalized training in integrative medicine

*Corresponding author 503-806-6900 503-227-3750 nvasilevsky@ncnm.edu. 
research. This program was designed to go beyond the ability to read research studies. Instead, students are trained with the skills necessary to both evaluate and conduct research within CIM. As of 2014, there are currently 52 students enrolled in the MSiMR program, 42 of whom are concurrently completing a Naturopathic Doctorate (ND) or Master of Science in Oriental Medicine (MSOM) degree. To date, nine students have graduated from the program.

Prior to the initiation of the MSiMR program, students had the opportunity to participate in research projects as part of an independent study. Nearly $20 \%$ of the medical students at NCNM (100 students) were involved in research at some level. Some students completed master's level work, but did not earn a degree. During this period of time, the education was not consistent, and students were not provided a formal curriculum. To determine the level of interest in starting the formal master's research training program, faculty and students at NCNM were surveyed and interviewed. Faculty responded favorably when asked if they were interested in improving the research curriculum at NCNM. Students expressed a similar interest, emphasizing a need for more formal training in basic laboratory methodology to better assess natural medicine's mechanisms of action and medical literature. As a result, the Introduction to Laboratory Methods course at NCNM was developed and subsequently required within the MSiMR program and is offered to students at all levels during their training. The course was designed to introduce students to the essential skills for scientific bench research, to aid in understanding the scientific process, and to expose them to methods that relate to clinical practice. Here we describe the curriculum development for this course.

\section{Course objectives}

The objective of the Introduction to Laboratory Methods course is to train students in basic laboratory skills, thereby enhancing their ability to design research studies that investigate CIM modalities. The outcome of the course is to equip students to: understand and adhere to basic lab safety requirements, comprehend and perform basic laboratory techniques (i.e. pipetting, making solutions, centrifugation, fundamental laboratory mathematics and data management), and understand the principles of sophisticated laboratory methods such as cell culture, flow cytometry, enzyme-linked immunosorbant assay (ELISA), polymerase chain reaction (PCR), psychophysiology (the study of correlations between the mind, behavior, and bodily mechanisms) (http://medical-dictionary.thefreedictionary.com/ psychophysiology), and dietary analysis. The class is specifically tailored to students in the MSiMR program and is intended to familiarize them with laboratory techniques they could use in their research projects. While there is a broad spectrum of topics that could be included, we tailored the specific topics to the types of assays that could be performed in the Helfgott laboratory or in collaboration with other laboratories in the area.

\section{Course content}

The Introduction to Laboratory Methods curriculum includes fundamental laboratory skills, conceptual understanding of common laboratory techniques, data analysis, and data management. In particular, the laboratory skills focus on safety, pipetting, using routine lab 
equipment, making solutions, and universal calculations found in experimental design. An outline of a sample course schedule is shown in Table 1, for both the 12-week and 8-week terms. Other topics include introduction to cell culture, ELISA, flow cytometry, and DNA technologies, such as polymerase chain reaction (PCR), to facilitate improved study design and literature comprehension. Future curriculum development is being implemented to include an introduction to the concepts and uses of high performance liquid chromatography (HPLC).

The Introduction to Laboratory Methods course is taught for one two-hour weekly session over 12 weeks. During the summer term, the course is condensed to accommodate the shortened academic term, meeting for three hours over eight weeks. No class is held in the final two weeks of the course (one week in the summer) to comply with NCNM academic policy and to allow students to complete a take-home final examination. The class size is limited to ten students, permitting both personalized interactions with the instructor and small-group learning exercises. In order to provide access to laboratory equipment, the course is held in Helfgott's educational laboratory, which is equipped with 4 lab bays, pipettors, centrifuges, a pH meter, a scale, and more specialized instrumentation including a light microscope, flow cytometer, tissue culture hood, absorbance microplate reader, and a high performance liquid chromatography (HPLC) system.

Guest lecturers from laboratories in the Portland metropolitan area expose students to diverse research opportunities that are available. When possible, field trips are taken to provide students with a first-hand understanding of the research methodology and facilities required to conduct large-scale clinical trials and high-volume clinical assays. For this course, students visit both a bionutrition laboratory and a large-scale clinical testing facility. During the bionutrition field trip, students learn about the studies and methods that are implemented in the facility, and they spend time in a research kitchen where they prepare nutritional shakes. The instructor guides them through the steps of designing a recipe for the shakes based on specific macronutrient ratios, and then the students taste-test the shakes. This gives students exposure to the research methodology involved in bionutrition studies. The second field trip takes students to a large-scale clinical testing facility. This field trip is intended to facilitate an understanding of both the technology underlying clinical assays used in routine clinical practice and how to collaborate with high-throughput clinical laboratories. During this field trip, the students learn about the company's diagnostic tests and research interests from the company's President, they share their own research project ideas, and they are given a tour of the clinical testing lab, where large-scale clinical assays are performed, such as ELISAs, mass-spectrometry, and liquid chromatography-gas chromatography (LC-GC).

The final component to teaching basic laboratory methodology is record keeping and data management. While the once prolific spiral bound, carbon copy paper lab notebooks are being replaced by their electronic counterparts [6], the fundamental aspects of data management remain. In the course, we discuss methods for keeping a good laboratory notebook, reinforcing the scientific method and teaching important organizational skills including how to track research resources and experimental details. Students are reminded that the scientific foundation is dependent upon the reproducibility of previously employed 
methods and techniques, emphasizing the need for thorough and complete documentation throughout an experiment. In addition, specific attention is placed on best practices for data management, including how to track computer files, how to back-up data, how to apply metadata and data standards, and how to share data.

\section{Teaching strategies}

The format of the class includes lecture presentations, interactive laboratory-based exercises and facilitated discussions. The objective is to introduce the students to basic concepts and theories involving scientific methodology, rudimentary laboratory techniques, common terminology and issues associated with study design. A typical class module begins with a lecture on a specific laboratory topic (approximately 45 minutes), followed by one to two lab exercises to demonstrate principles discussed in the lecture (approximately 1.25-2 hours). The use of a textbook is replaced with reading assignments including current news articles with laboratory relevance or the primary literature in an attempt to apply the course content to real-life situations.

The initial module is an introduction to fundamental laboratory techniques, for which a novel exercise named the Berry Exercise was developed. This exercise takes students through the steps involved in making berry jam using laboratory equipment including scales, centrifuges, pipettors and $\mathrm{pH}$ meters instead of culinary equipment. This interactive and engaging module serves to set the foundation for all future modules in which these rudimentary skills are frequently used.

The cell culture module is designed to give students an overview of the application, principles, and techniques of cell culture. Students begin by reflecting on the utility of growing human cells in a lab; this approach gives students an opportunity to contemplate the use of cell culture before being introduced to the material. The student's answers are then incorporated into the lecture as each new topic is introduced. The lecture portion of the class covers a broad range of topics, from the real-world applications of cell culture and cell line derivation to more technical topics like growth medium composition and how to prepare cell culture media. Laboratory exercises expose students to a variety of cell culture-related activities. Students rotate through stations where they calculate dilutions, prepare cell culture media from stock solutions, and assess the $\mathrm{pH}$ of fresh versus aged growth medium.

Students also examine cell morphology using phase-contrast microscopy, count cells using a hemacytometer, and assess viability by staining cells with a viability dye.

The objective of the flow cytometry section is to introduce the concept of flow cytometry, its technical aspects, considerations and use, while providing hands-on experience in experimental design and protocol execution. To this end, a combination of a lecture, tutorial videos, and active learning activities are used. A PowerPoint lecture introducing the topic is complemented by in class or at-home tutorial videos that were developed by BD Biosciences (http://www.bdbiosciences.com/services/training/itf_launch.jsp). Students then complete individual or group exercises that involve planning a flow cytometry experiment. For example, the students are asked to design an experiment with a specific number of samples to be analyzed for specific proteins. They determine which antibodies to use and calculate 
the appropriate dilutions which requires them to apply their laboratory math skills. To emphasize the importance of good record keeping, students are required to report the details about their resources, such as the antibody catalog number and host organism from which the antibody was derived. Next, the students use their own protocol to prepare samples, and with the assistance of the instructor, run their samples through the flow cytometer. At the completion of the experiment, the students are asked to read a previously published paper using the same methodology (in this example it is [7]), after which they analyze and interpret the data that are presented.

A very similar format is used in each of the other modules with some modifications when commercial tutorial kits are available. For the ELISA module, a lecture is followed by a lab assay, using the BioRad ELISA Explorer Kit (cat \#166-2400EDU). This kit was selected because it provides all of the necessary reagents and an accelerated version of an ELISA experiment. The students test a specific hypothesis, such as determining if their fellow students have been exposed to a hypothetical virus, which can be tested in approximately one hour using the kit. As with the other modules, this is followed with paper discussion and data interpretation exercises for a comprehensive understanding of the ELISA methodology and applicability.

As the research projects in the MSiMR program primarily focus on clinical research, the students are not exposed to animal research during their training. However, animal research plays a central role in translational research, so the curriculum includes a component surrounding the use of animal models in biomedical research. Students are introduced to the history of animals in research, asked to brainstorm animal models the students are aware of, and then the use of various animal models is introduced including transgenic models bred onto mouse, rat or zebrafish strains, or the use of primates for pre-clinical research. The ethical issues surrounding animal research are discussed and the regulations (http:// awic.nal.usda.gov/government-and-professional-resources/federal-laws/animal-welfare-act) and guidelines for reporting use of animals in research are reviewed (http://jp.physoc.org/ content/588/14/2519.full.pdf $+\mathrm{html}$ ). Finally, the students are asked to debate the advantages and alternatives for the use of animals in research.

In teaching basic laboratory techniques, it is necessary to convey how these fundamental methods apply on a broader scale, including biomedical literature interpretation and evidence-based medicine use in clinical practice. When discussing specific methodologies, such as apoptosis assays, it was important to step back and explain not only what the assay is, but also why would it be done, and how it can answer specific research questions. Finding examples from current news articles or the current literature helped relate these specific methods to studies of interest within CIM.

On the final day of the course, the material is reviewed in a Jeopardy game format using templates readily available online. Topics include Laboratory Mathematics, Basic Laboratory Skills, Flow Cytometry, ELISA and Experimental Methods in an attempt to address all components of the course. Students are separated into two teams, with the questions getting progressively harder as the monetary categories increase. To review the bench skills taught in the course, students participate in a lab practicum. Stations are set up 
around the lab, with instructions at each station to perform various lab activities, such as balancing a centrifuge or testing pipetting accuracy by measuring the weight of water.

Student assessment is based on attendance, punctuality, and take-home exams. Initially only a final exam was given at the end of the course, but in more recent course offerings, a midterm exam was incorporated into the curriculum to allow for increased opportunities for assessment. The take-home exams are open resource and are comprised of questions pertaining to all of the course modules to assess comprehension. Examination of concepts learned during the field trips is also included. The open resource format was selected over a timed in-class summative assessment. While the latter may have given a more objective assessment of the student's mastery of the course, it was felt that the take home exam would assess the students' conceptual understanding of the material and ability to find, retrieve, and apply the information that was discussed in the course. This format was thought to better represent a real-life laboratory environment, where students have access to the Internet and other available resources, making memorization of specific concepts unnecessary. Analysis of the average of final grades across all students $(n=38)$ is 3.7 (on a 4.0 scale), suggesting most students mastered the course. While the graded assessment is based only on the conceptual understanding, students who go on to incorporate lab-based methods into their research projects undergo more formal one-on-one training. For example, extensive cell culture training is done during 2-hour sessions for 10 weeks, where they begin with basic calculations for preparing cell culture media and finish with culturing a cell line in vitro. The Introduction to Laboratory Methods course provides a foundation for the more extensive bench research training for the MSiMR students who undertake their own bench research projects.

\section{Curriculum Effectiveness}

Students were surveyed at the beginning and end of the course to assess their understanding and confidence in performing laboratory-based experiments and to determine if this course augmented it. After providing informed consent, students answered ten questions based on a $1-10$ scale, with $1=$ strongly disagree and $10=$ strongly agree. The survey results were compiled from 18 students over 3 terms, and the averages and standard deviations were calculated in Microsoft Excel (v.14.4.4) and the p-value was calculated using a TTEST. The survey results revealed that students had an increased understanding of basic laboratory methodology $(\mathrm{p}=0.001)$ and significantly increased familiarity with lab techniques (ELISA, $\mathrm{p}=0.00004$, Flow cytometry, $\mathrm{p}=0.00005)$ and terminology $(\mathrm{p}=0.0001)$ after completion of the course (Table 2). In the pre-class survey, several students indicated they felt comfortable with performing lab work $(n=7)$, using common equipment $(n=6)$ and doing basic lab math $(\mathrm{n}=6)$, suggesting they had prior lab experience (students answering 8 or above on questions 1-3 in the survey in Table II). To determine if this course augmented their skills, a subanalysis was done with this subset. For all three responses, there was not a significant difference between their responses at the start of the course or after completion, which suggests the course did not augment the basic lab skills for students with prior lab experience. The entire datasets are provided as Supplementary Tables 1 and 2. Overall, this course did appear to augment the students' familiarity and understanding of basic lab skills, 
and other programs that have initiated research methods programs have also reported similar results $[3,8]$.

\section{Challenges and Successes}

With the development of any new course, there are always challenges and successes. One particular challenge was the time constraints associated with the short duration of the course (20 hours of classroom time). Limited time made setting up studies, implementing them, and obtaining results quite challenging. An additional obstacle was foreseeing and planning the time necessary for students to perform laboratory exercises, as students complete the tasks at different rates based upon prior experience levels. At times, it was more effective to allow the students to perform the laboratory components at the beginning of the module, and then review and lecture on relevant topics after the lab activities were finished to ensure adequate time for experiment completion. Students with prior lab experience were often able to finish the lab activities more rapidly, so these students performed additional exercises or were asked to assist other students in order to keep them engaged. To troubleshoot, we found it beneficial for the instructor to practice the exercise prior to performing it in class. We found that an appropriate estimate for a student researcher performing a new technique for the first time was approximately two to three times longer than an experienced researcher.

Students often struggled with laboratory calculations. The ability to select basic equations and complete calculations when making solutions or setting up assays is a critical skill that requires continued practice. Thus, we introduced basic laboratory equations and examples at the beginning of the course and then had the students perform exercises throughout the duration of the course. The goal of incorporating it throughout was to provide a foundation that could be applied to future research endeavors and help students gain confidence in performing laboratory calculations.

The laboratory field trips were especially well-received. The exposure to techniques and investigations outside of the NCNM facility expanded the students' knowledge of research methodology and provided valuable networking opportunities. In addition, mentorship opportunities were offered to help guide students with their individual research interests. The course was designed to expose them to basic laboratory skills; the mentorship component was intended to facilitate further progression with the individual's research projects and refine their design skills. Overall, the Introduction to Laboratory Methods course at NCNM has been viewed as a success, and we continue to refine and enhance the curriculum with each new term of students and their specific needs.

\section{Supplementary Material}

Refer to Web version on PubMed Central for supplementary material.

\section{Acknowledgements}

This work was supported by NIH-NCCIH grant number 5R25AT002878. 


\section{References}

1. Briggs JP, Killen J. Perspectives on complementary and alternative medicine research. JAMA: the journal of the American Medical Association. 2013; 310(7):691-2. [PubMed: 23990064]

2. Kreitzer MJ, Sierpina V, Maiers M, Delagran L, Baldwin L, Evans R, Chase M. Ways of knowing: integrating research into CAM education and holism into conventional health professional education. Explore. 2008; 4(4):278-81. [PubMed: 18602624]

3. Lasater K, Salanti S, Fleishman S, Coletto J, Jin H, Lore R, Hammerschlag R. Learning activities to enhance research literacy in a CAM college curriculum. Alternative therapies in health and medicine. 2009; 15(4):46-54. [PubMed: 19623832]

4. Lee MY, Benn R, Wimsatt L, Cornman J, Hedgecock J, Gerik S, Zeller J, Kreitzer MJ, Allweiss P, Finklestein C, Haramati A. Integrating complementary and alternative medicine instruction into health professions education: organizational and instructional strategies. Academic medicine: journal of the Association of American Medical Colleges. 2007; 82(10):939-45. [PubMed: 17895652]

5. Nedrow AR, Heitkemper M, Frenkel M, Mann D, Wayne P, Hughes E. Collaborations between allopathic and complementary and alternative medicine health professionals: four initiatives. Academic medicine: journal of the Association of American Medical Colleges. 2007; 82(10):962-6. [PubMed: 17895657]

6. Butler D. Electronic notebooks: a new leaf. Nature. 2005; 436(7047):20-1. [PubMed: 16001034]

7. Mikolai J, Erlandsen A, Murison A, Brown KA, Gregory WL, Raman-Caplan P, Zwickey HL. In vivo effects of Ashwagandha (Withania somnifera) extract on the activation of lymphocytes. Journal of alternative and complementary medicine. 2009; 15(4):423-30. [PubMed: 19388865]

8. Zick SM, Benn R. Bridging CAM practice and research: teaching CAM practitioners about research methodology. Alternative therapies in health and medicine. 2004; 10(3):50-6. [PubMed: 15154153] 
Table I

Sample class schedule for either the 12-week or 8-week term.

\begin{tabular}{|l|l|l|}
\hline & Topic & Topic \\
\hline Week & 12-Week Term & 8-Week Term \\
\hline 1 & Introduction & Introduction \\
\hline 2 & Basic lab skills, pipetting, making solutions & Basic lab skills, pipetting, making solutions, Berry exercise \\
\hline 3 & Berry exercise & Cell culture \\
\hline 4 & Cell culture & ELISA, Psychophysiology \\
\hline 5 & Bionutrition field trip & Bionutrition guest lecture or field trip \\
\hline 6 & Flow cytometry & Flow cytometry, DNA technologies \\
\hline 7 & DNA technologies & Animal models, Jeopardy \\
\hline 8 & Psychophysiology, Animal models & Take home final exam \\
\hline 9 & ELISA & \\
\hline 10 & Jeopardy & \\
\hline 11 & No class & \\
\hline 12 & Take home final exam & \\
\hline
\end{tabular}




\section{Table II}

Student responses to questionnaire regarding their impressions of the course.

\begin{tabular}{|l|l|l|l|}
\hline Question & Pre-class (average) & Post-class (average) & p-value \\
\hline I am familiar with methods and results that I read about in research papers & 6.5 & 8.2 & 0.001 \\
\hline I understand basic laboratory terminology & 6.6 & 8.9 & 0.0001 \\
\hline I am comfortable performing lab work & 6.5 & 7.3 & 0.17 \\
\hline $\begin{array}{l}\text { I am comfortable using common laboratory equipment (centrifuge, pH meter, } \\
\text { pipettes, etc) }\end{array}$ & 6.2 & 8.4 & 0.01 \\
\hline I understand the concepts of ELISA & 4.1 & 8.1 & 0.00004 \\
\hline I understand the concepts of flow cytometry & 4.1 & 7.7 & 0.00005 \\
\hline I am confident doing basic lab math (calculating percentages, dilutions, etc.) & 7.4 & 7.9 & 0.27 \\
\hline I am familiar with what is available at NCNM to tailor research projects & 5.2 & 8.6 & 0.0000003 \\
\hline $\begin{array}{l}\text { I am comfortable designing and setting up experiments (such as choosing } \\
\text { controls, determining the right assay to address a question) }\end{array}$ & 5.7 & 7.2 & 0.02 \\
\hline I have an understanding of how, or if, the lab plays a role my research project & 7.0 & 8.6 & 0.01 \\
\hline
\end{tabular}

